\section{Dimensión pedagógica de la Educación Virtual: \\ Una reflexión pendiente}

Pedagogic dimension of

Virtual Education,

A pending reflection.

\section{Dimensão pedagógica da educação virtua Uma reflexão nao resolvida}

\section{Ana Claudia Rozo Sandoval ${ }^{*}$}

* Máster en Ciencia, Tecnología y Sociedad: Cultura y Comunicación en Ciencia y Tecnología, Universidad de Salamanca, España. Coordinadora línea de investigación institucional Pedagogía, Medios y Mediaciones, Fundación Universitaria Los Libertadores. Bogotá, Colombia.

Correo electrónico: anclarozo@gmail.com.

\section{Resumen}

Este artículo se desarrolla en el en el marco de la investigación "Educación Virtual en las Instituciones de Educación Superior, una propuesta interpretativa". El trabajo da cuenta de una revisión de veinte investigaciones realizadas en Iberoamérica, en las cuales se aborda la comprensión de lo pedagógico desde tres elementos de análisis: los entornos virtuales de aprendizaje; las plataformas y otras tensiones en educación virtual; los actores de la educación virtual y, la perspectiva cultural de la Educación Virtual.

\section{Palabras Clave}

Educación virtual, pedagogía, sujetos virtuales, plataformas, perspectiva cultural de la educación virtual.

\section{Abstract}

This article is developed in the frame of the research project: "Virtual Education at Higher Education Institutions, an interpretative proposal". This piece of work comes to a checking out of twenty research projects done in Latin America, which comprehension of the pedagogic matter is dealt with three different aspects of analysis: learning virtual environments, platforms and other tensions in virtual education; actors of virtual education and, the cultural perspective of virtual education.

\section{Key words}

Virtual education, pedagogy, virtual individuals, platforms, the cultural perspective of virtual education.

\section{Resumo}

Este artigo é producto da pesquisa Educação virtual em Instituições de ensino superior: uma proposta interpretativa, que revisa vinte pesquisas levadas a cabo na Ibero-América, nas quais se aborda a compreensão do pedagógico a partir de três elementos de análise: ambientes virtuais de aprendizagem, plataformas e tensões na educação virtual; atores da educação virtual; e perspectiva cultural da educação virtual.

\section{Palavras-chave}

Educação virtual, pedagogia, sujetos virtuais, plataformas, perspectiva cultural da educação virtual.

Fecha de recepción: 21 de septiembre de 2009 Fecha de aprobación: 28 de noviembre de 2009

Investigación realizada por el grupo Comunicación, Cultura y Tecnología de la Fundación Universitaria Los Libertadores que pretende responder a la pregunta de qué es lo virtual de la educación virtual. El problema se analiza desde cuatro dimensiones: filosófica, tecnológica, comunicativa y pedagógica, en atención de lo político como contexto.

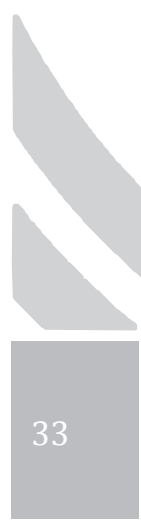


sentido se entrelazan íntimamente con lo que la sociedad es y con lo que sus actores proyectan ser" (Benítez, 2000, p.6). El impacto de las Tecnologías de Información y Comunicación, las modificaciones en los modos de producción, asociados a una idea y enfoque de desarrollo y las demandas del sector productivo en relación con una fuerza de empleo determinada ${ }^{3}$, entre otros aspectos, delimitan fines, sentidos y modelos educativos que hoy hacen carrera sin más análisis, ni cuestionamiento.

Los conceptos de sociedad de la información y de sociedad del conocimiento (Castells) se soportan en la idea de que la información y el conocimiento adquieren valor y se constituyen en uno de los fundamentos de la sociedad -esta concepción también tiene contradictores que pueden ser rastreados en la literatura acerca del tema-. Este aspecto exige orientar programas de formación que apunten a identificar las modificaciones que tales concepciones generan en los procesos de producción, de generación de riqueza y de la idea de desarrollo. Sin embargo, las miradas centradas en los artefactos tecnológicos, en las estructuras de conexión de las redes de información y en los diseños instrumentales resultan insuficientes para comprender y dar respuesta a este nuevo ordenamiento:

[...] en esta bifurcación empresarial al valor asignado al conocimiento, se reduce y cosifica los elementos más profundos del pensamiento, y con ello también se evidencia la superficialidad en que es considerada la educación misma. De hecho, los falsos profetas, tecnócratas e ignorantes se ven incapaces de construir una delimitación conceptual aceptable para la educación digital si es que fuera posible su existencia, creen que al calificar como "tradicional" a los sistemas presenciales, establecen una fractura epistemológica que sostenga a la educación telemática (Pérez, 2003, p. 9).

\section{Desafíos de la dimensión pedagógica en la educación virtual}

En la comprensión de una educación virtual atravesada por dinámicas distintas a las de la educación presencial - y no como simulación o reproducción de las aulas de clase, de sus contenidos y de sus actores - y construida desde el entendido de lo virtual como "habitar la red" — con sus implicaciones directas en la configuración de identidades y

3 El eje gravitacional del empleo, analizado por Peter Drucker (1989), ha significado para algunas sociedades el traslado de lo industrial, manual, administrativo hacia los trabajadores del conocimiento, lo cual se ha hecho posible con el desarrollo, la implementación y el uso de las TIC. subjetividades, de lenguajes y formas de comunicación, de otras relaciones con la información y con el conocimiento lo pedagógico- supone, en esencia, una conexión con la cultura, el poder y los sujetos en una constante tensión que obliga a despojar las certezas, a reestructurar (de construir y construir) las cadenas de conceptos y a transitar por senderos de posibilidad, a modo de Lévy.

En la dimensión pedagógica de la E.V. la pregunta por quienes intervienen en el acto educativo desde sus múltiples dimensiones - histórica, política y cultural, en condición y capacidad de enseñar y aprender, de ser autor y lector que participan e interactúan en espacios tecnológicos y mediáticos a través de los cuales se vehiculiza el proceso educativo-quebranta los enfoques que procuran elaboraciones de contenidos uniformes y estáticos puestos en plataformas de e-learning, desde los cuales se pretende una "educación virtual" homogénea, global y universal con estándares internacionales de calidad.

En la literatura revisada, la orientación homogenizante propone una tendencia socializadora de lo pedagógico al considerar las posibilidades de aprendizaje de los estudiantes desde distintos referentes. Sin embargo, en la práctica se evidencia el diseño e implementación de procesos instruccionales, soportados en el diseño y en la puesta en plataformas de materiales y recursos, que trasladan las prácticas de aula y las concepciones de lo educativo, lo comunicativo del escenario real al entorno de la red.

La apuesta de esta dimensión encuentra que "la pedagogía debe entenderse no solo como productora de conocimiento, sino también de sujetos políticos, en ese sentido la comprensión del "otro", del distinto, del diferente exige aproximaciones al diseño de propuestas formativas en la distancia que reconozcan esta individualidad" (Ortega, 2008. p. 65).

Desde tal comprensión, se hace indispensable atender diversos asuntos que trascienden la idea generalizada de "modelos pedagógicos" estáticos y rígidos, valorados con criterios de eficacia y eficiencia: las modificaciones que se dan en la relación información-conocimiento en estos escenarios, los fenómenos espacio-temporales que quiebran la noción de presencialidad, las consideraciones de las identidades individuales y colectivas, los procesos de construcción de conocimiento individual y colectivo, la elaboración de currículos flexibles y los criterios de evaluación como procesos que den cuenta tanto de los aprendizajes disciplinares como de la formación de hombres y mujeres en su dimensión humanista en la perspectiva de los nue- 
vos ordenamientos de creación y construcción de los procesos comunicativos-educativos desde donde se cuestionan los esquemas horizontales y unidireccionales. Esta perspectiva demanda la deconstrucción y la construcción como constante de los aspectos que tensionan permanentemente la E. V.

\section{Revisión Documental}

El lugar desde el cual se teje la dimensión pedagógica sirve de referente para explorar las elaboraciones que, desde los trabajos de investigación analizados, permiten dibujar el estado de la investigación en esta materia. Análisis que se centró en tres focos de observación:

- Relaciones entre quienes intervienen en el acto pedagógico. Se busca así identificar los actores que participan en el entorno mediado tecnológicamente y desde el cual se construyen otras subjetividades, otras formas de comunicación, otros roles de maestros y estudiantes y desde donde emergen otros actores que construyen el proceso.

- Información-conocimiento. Relación que hace explícita las concepciones que fundamentan lo educativo y lo pedagógico.

- Ambiente educativo. La comprensión de E. V. asociada al uso de plataformas pone de relieve el diseño de Ambientes Virtuales de Aprendizaje (AVA) o de Entornos Virtuales de Aprendizaje (EVA) en donde se supone tiene lugar el proceso educativo. Aquí se hace importante considerar la relación con el contexto, las diferencias culturales, los estilos cognitivos y los estilos de aprendizaje, entre otros asuntos que van configurando una manera de asumir lo pedagógico en espacios mediados tecnológicamente. Aspectos referidos más a un ambiente educativo que a un ambiente de aprendizaje.

Se revisaron veinte documentos con información de resultados, avances o reflexiones acerca de investigaciones en curso o terminadas. La variedad de los trabajos permitió realizar una caracterización inicial, en función de los temas centrales abordados por los investigadores:

- De los entornos virtuales de aprendizaje, las plataformas y otras tensiones en educación virtual.

- Los actores de la educación virtual: ¿sujetos virtuales?, identidades perdidas.

- Perspectiva cultural de la educación virtual.
Las investigaciones se ubicaron de acuerdo con el énfasis desde el cual se desarrollaron los trabajos mismos -esto no significa que no se relacionen entre ellos, aunque no todos toman como referente a quienes participan en el proceso educativo-. Una vez hecha esta salvedad, vale reconocer que algunas experiencias permitieron aproximaciones distintas a los trabajos centrados en enfoques instrumentales de la tecnología y la educación; se cuestionan así los diseños universales y estáticos de los OVAS, EVAS, AVAS y demás propuestas que indagan por otras formas de aprender, de producir y de apropiar conocimiento en el entorno de las redes; además y, de manera contundente, se hace explícito que la mera adopción de Nuevas Tecnologías no significa directamente innovación, y aún menos la ruta hacia la calidad.

\section{De los entornos virtuales de aprendizaje, las plataformas y otras tensiones en Educación Virtual}

Los documentos revisados en esta categoría ${ }^{4}$ permiten inferir que aún se registran investigaciones centradas en diseños más eficientes para instrumentalizar el proceso educativo, esto es delegar a los medios -textos, vídeos, audios, multimedios, hipertextos, etc.- la responsabilidad de la formación. Sin embargo, se reconoce un desplazamiento interesante hacia trabajos que se ocupan por indagar acerca de procesos de aprendizaje en la red; es decir, preguntarse por el tipo de enseñanza pertinente en los entornos mediados tecnológicamente, además de reflexionar de manera sistemática acerca del papel de los docentes en función de su compromiso con la educación y de formular y llevar a cabo propuestas innovadoras para la formación de los maestros en estos ambientes que, dicho sea de paso, en su mayoría han sido formados en y para la presencialidad.

La investigación de Onrubia y el trabajo doctoral de Pérez interrogan la instrumentalización de la educación y plantean desde sus análisis alternativas importantes que ponen el acento en lo educativo: consideran los contextos, el cómo se aprende, las múltiples dimensiones del sujeto en la red y las interacciones que allí tienen lugar. Onrubia (2005), con base en un enfoque constructivista-sociocultural, propone tres estrategias: actividad conjunta, ayuda pedagógica y construcción del conocimiento; desde

4 Pérez, 2003; Onrubia, 2005; Correa, 2005; Torres, 2005; Silva, 2005; Gallardo, Torrandell, Negre, 2005; Salinas, Negre, Gallardo, 2006 
“...el uso de Tecnología de Información y Comunicación en sí mismo no resuelve ningún problema educativo, ni mejora la calidad, ni representa ninguna innovación; en especial si, asociado a ello, no existe una comprensión de las transformaciones estructurales que se dan en la red y, en consecuencia, de propuestas pedagógicas que atiendan tales desafíos."

allí fundamenta su apuesta por la construcción de conocimiento y, en consecuencia, refiere las implicaciones que esta perspectiva tiene en el diseño y en la evaluación de los entornos. Por ende, interroga las propuestas universales, permanentes y estáticas de los Objetos Virtuales de Aprendizaje (OVA); pone de relieve, además, las relaciones que se tejen entre docente y estudiantes, aspecto que considera como factor decisorio en la construcción de conocimiento y que sirve de andamiaje en el proceso. Ante la heterogeneidad de los estudiantes expone unas preguntas constantes: ¿qué, por qué y para qué aprenden los estudiantes?

Desde la Universidad Complutense, el trabajo de Pérez acerca de plataformas digitales y sus fracturas pedagógicas hace explícito cómo los sistemas educativos atienden las demandas del sistema productivo y las fracturas del conocimiento generadas por el "eje gravitacional del empleo" (P. Drucker 1989); aspectos que ocasionan modificaciones en las demandas de formación que el sector productivo hace a la Universidad. Después de analizar 105 cursos virtuales, muestra las debilidades de los enfoques educativos que, a través de plataformas, delegan la responsabilidad con el conocimiento a diseños sofisticados y atractivos, sin tener en cuenta los quiebres o las fracturas sociales, culturales y comunicativas que inciden directamente en la educación y que en su estudio se identifican como fracturas del conocimiento, de la evolución y de la educación. Cada uno de estos quiebres da cuenta de los factores que afectan la reconfiguración de los propósitos y modelos educativos: demandas de "calidad" y de formación y transformaciones sustantivas en los procesos y modos de comunicación y transmisión de saberes - de la oralidad a la escritura, de la imagen en formatos audiovisuales al manejo de códigos informacionales de la informática-. En estos tránsitos, las relaciones entre información y conocimiento van demarcando unos modos de asumir lo educativo asociados a la asimilación, la reproducción o la producción de conocimiento.

Los estudios de Gallardo, Torrandell, Negre (2005) y Salinas, Negre y Gallardo (2006), del grupo de Tecnología Educativa de la Universidad de las Islas Baleares, se centran en analizar los componentes de los modelos didácticos en la educación superior en los entornos virtuales y en formular una propuesta de modelos didácticos en esta materia. Los investigadores abordan la indagación desde los componentes de Ambientes de Aprendizaje, a través de los cuales reconocen tres dimensiones: pedagógica, tecnológica y organizativa. En los resultados del primer estudio dan cuenta de las interrelaciones que se tejen desde estas dimensiones $\mathrm{y}$, a partir del rastreo de estudios previos, caracterizan los diferentes componentes de cada dimensión. La pregunta que subyace a este trabajo se relaciona con la identificación de los componentes que determinan el paso de unos modelos de enseñanza aprendizaje a otros. En la formulación de la propuesta del modelo, y luego del proceso de investigación, el grupo afirma: "Partiendo de la idea de que probablemente no se requiera un nuevo modelo para explicar los procesos de enseñanza-aprendizaje en entornos virtuales, al no encontrarse en este ámbito un cambio de paradigma que los justifique, se ha pretendido resituar y redimensionar los elementos que aparecen en los modelos didácticos clásicos, poniéndolos a consideración de los expertos junto a elementos que aparecen en la literatura actual referida al uso de los entornos virtuales" (2006, p.16).

Los resultados de estos trabajos, en su mayoría de corte cualitativo con una marcada tendencia a indagar por los procesos de aprendizaje en las plataformas y a identificar los desajustes evidentes cuando las propuestas centran su desarrollo en las plataformas y los diseños, enfatizan en el señalamiento de que el uso de Tecnología de Información y Comunicación en sí mismo no resuelve ningún problema educativo, ni mejora la calidad, ni representa ninguna innovación; en especial si, asociado a ello, no existe una comprensión de las transformaciones estructurales que se dan en la red y, en consecuencia, de propuestas pedagógicas que atiendan tales desafíos. 


\section{Los actores de la Educación Virtual ¿sujetos virtuales? identidades perdidas}

En esta categoría se ubicó el mayor número de trabajos analizados desde la dimensión pedagógica ${ }^{5}$. Trabajos donde parecería tener gran relevancia la idea de un "sujeto virtual", configurado y revestido de sentido en función de su relación con el conocimiento. Desde este enfoque adquieren nuevos sentidos las preguntas relacionadas con: la forma de aprender en la red, las relaciones y la forma de hacer visible el sujeto del mundo material (real) en el entorno digital, la limitación de la enseñanza a los materiales puestos en el "ambiente virtual", el papel del maestro, del tutor o del acompañante en el proceso de construcción de conocimiento, la forma como se dan los procesos comunicativos y las interacciones en este entorno y su implicación con el aprendizaje.

Se registran análisis que refieren la importancia de los aspectos sociales en las propuestas educativas orientadas al mundo de la red (Ross, 2004), en la comprensión de que el sujeto partícipe de estos procesos formativos vive y se desarrolla en entornos sociales y culturales específicos. Desde esta orientación, la reflexión del maestro acerca de su práctica como tutor da origen a otra línea de trabajos que interroga el rol del maestro tutor en estos escenarios (Martínez, Briones, 2007; Sarle, Rodríguez, 2008). Otros trabajos sugieren el diseño o la adaptación de ambientes digitales con base en enfoques socio-constructivistas, centrados en el estudiante (Del Moral, Villalustre, 2005; Mauri, Colomina, 2007), como factores que aportan al mejoramiento educativo.

El "sujeto virtual" asociado a la educación hace explícita la crisis de identidad de los "habitantes del entorno red". Esto ocurre, de manera especial, en las apuestas que trascienden el concepto de virtualidad como simulación y se orientan desde la comprensión de la complejidad del trabajo en la red; atienden las múltiples subjetividades, los lenguajes, las interacciones, las nuevas relaciones con la información y las otras formas de producir conocimiento, unidas a lo virtual como escenario de posibilidady, en particular, de la educación en entornos digitales ${ }^{6}$.

5 Mungaray (2005; 2007); Coll, Mauri, Onrubia (2006); Flores (2006); Ilvento, Martínez (2007).

6 Títulos como "Encontrando al profesor virtual Acerca(r) de la construcción de imaginarios identitarios en el tutor virtual" permiten una aproximación a esta idea.
De acuerdo con Mungaray,

[...] este sujeto virtual, revisado desde las propuestas paradigmáticas, pretende ser conformado desde otras dimensiones asociadas al ciberespacio y al cibertiempo. Esta atribución del sujeto, surge desde un planteamiento situacional que reclama un proceso de resolución del sujeto frente al mundo nuevo; esto incluye no sólo la definición etimológica previa y esperada, sino también las discusiones colaterales donde lo virtual no es entendió ingenuamente en oposición a la realidad, sino como un planteamiento que se resuelve como potencial, posibilidades o espectro de diversificación frente al mundo que se conoce y al mundo "hiper" que se intuye (2005, p.5)

Se evidencian, entonces, tensiones entre la comprensión y la actuación en el mundo real y la forma de ser sujeto; entre las identidades y las subjetividades que se extienden en el entorno digital. En este escenario, de acuerdo con las investigaciones revisadas, la crisis de identidad del maestro se pone de presente alrededor de cuatro aspectos específicos:

a. La extensión de las formas de ser sujeto en el mundo material al entorno digital, el cual se reconoce como generador de grandes transformaciones.

b. La esencia del maestro formado en y para la presencialidad frente a un escenario digital donde se establecen tanto alteraciones en términos de interacciones como relaciones con la información y el conocimiento -ahora mediados tecnológicamente-, según se sugiere en Ilvento, Martínez (2007).

c. El diseño de los ambientes virtuales de aprendizaje, en los cuales los materiales - textos, vídeos, audios, hipertextos, multimedias, etc.- contienen la información necesaria para generar el aprendizaje en los estudiantes, lo cual ha llevado a que el papel del maestro se debata entre experto, diseñador de ambientes, tutor, acompañante, mediador y gestor, entre otros roles adjudicados, para permitir la autonomía del estudiante, quien hace posible su existencia (la del maestro) cuando se le demanda, se le interroga o se le requiere.

d. Las formas de enseñar, en función de modelos pedagógicos que orientan diferentes estructuras y actores. Actores que van desde la figura de expertos -responsables de la definición de contenidos, temas y actividades-, pasan por el rol de diseñadores, hasta llegar a ocupar el rango de tutores. Éstos pueden llegar a atender simultáneamente y de la misma manera -es decir, cinco o seis tutores responden a 100 ó 120 estudiantes como si fueran un solo tutor(Flores, 2006).

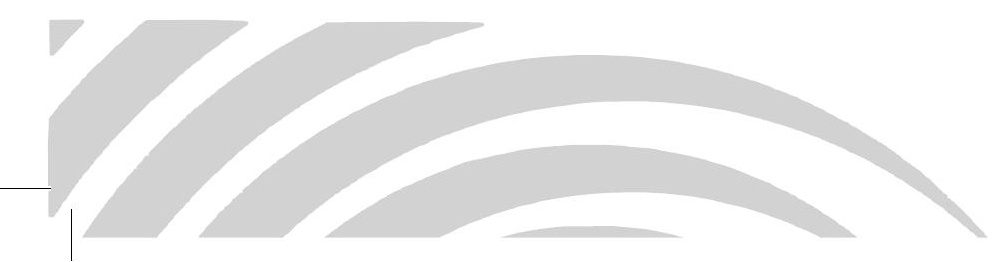


En el conjunto de trabajos, las temáticas que abordan la construcción de conocimiento en los escenarios mediados tecnológicamente se convierte en asunto de indagación permanente ${ }^{7}$. Desde la perspectiva del sujeto virtual de conocimiento, propuesta por Mungaray, se cuestionan las formas de construir conocimiento: "la discusión que seguimos se entreteje frente a los retos del flujo de información y a un cuestionamiento sobre si lo pertinente es mantener una estructura de conocimiento sólida para su análisis o si se tiende a formalizar una nueva forma de conocer que asimile la tensión derivada de incorporar la información dinámica y volátil. Es decir, documentando cómo se da el conocimiento bajo los requerimientos de una lógica estructural frente a una lógica hipertextual" (2005, p.9). De acuerdo con los resultados de la investigación, el reto propuesto en relación con la construcción de conocimiento legitima la importancia de integrar lenguajes, tecnologías y de relacionar conocimientos de la lógica formal, académica, con conocimientos marginales, no reconocidos por estas estructuras.

Los aportes del grupo de la $\mathrm{UOC}^{8}$ reconocen los ambientes híbridos -entorno digital y escenario presencial- como adecuados para la construcción de conocimiento, a partir de estrategias de resolución de problemas que procuran desarrollar habilidades de búsqueda, análisis e interpretación de información desde escenarios de aprendizaje colaborativos que también se implementan por parte de los docentes. Se reconoce como constante la reflexión y el análisis para el diseño de estrategias de formación que reconocen las particularidades de los estudiantes y sus intereses.

En relación con la identidad de los maestros en la red, los investigadores acuden a trabajos orientados desde la reflexión de la práctica del tutor ${ }^{9}$. Uno de los elementos recurrentes en los resultados se reconoce en el papel del tutor que, en los escenarios mediados tecnológicamente, orienta su trabajo a individuos integrados en un colectivo. Tal situación exige el diseño de estrategias que permitan atender a esta individualidad múltiple; el tutor traslada su práctica pedagógica expositiva a una práctica pedagógica estratégica, apoyada en proceso de trabajo colaborativo: "el profesor asociado a un saber colectivo que debe organizar y ayudar a compartir. En este sentido, el proceso de

7 Ros (2004); Del Moral, Martínez (2005); Coll, Mauri, Onrubia (2006); Mungaray (2005; 2007).

8 Coll, Mauri, Onrubia (2006).

9 Flores (2006); Ilvento, Martínez (2007); Martínez, Maris (2007); Sarlé, Rodríguez (2008) enseñanza y de aprendizaje se ve como un diálogo en colaboración entre elementos diversos" (Barbera citado en Ilvento, Martínez, 2007).

En relación con quienes participan del proceso de aprendizaje en el escenario digital los investigadores conceden un lugar importante a la idea de que los participantes que aprenden en los entornos mediados tecnológicamente no se hacen sujetos sólo en ese espacio $0^{10}$ : sus relaciones sociales y culturales en el mundo material les permiten configurar una manera de ser y estar en el espacio digital y, en consecuencia, de aprender en la "educación virtual". Los autores señalan que el enfoque socioconstructivista del aprendizaje en estos escenarios contribuye de manera significativa al mejoramiento de las prácticas educativas en educación virtual, donde la investigación, la reflexión y el análisis son constantes del proceso.

Las apuestas de trabajo, tanto de docentes, tutores e investigadores, como las orientadas a los estudiantes muestran un interés por el "trabajo colaborativo", "el aprendizaje en red", en el reconocimiento del valor, en los entornos digitales, adquieren las comunidades. Se considera primordial resaltar la concepción de autonomía que se propone en estos espacios, frente al interés por fortalecer la comunidad como los "individuos" que habitan la red.

\section{Perspectiva cultural de la Educación Virtual}

Los trabajos clasificados en esta sección orientan su análisis desde perspectivas que consideran la complejidad de los procesos culturales en relación con las transformaciones propias del entorno digital ${ }^{11}$. Así, preguntas por las identidades, por los lenguajes y por la imbricación de los escenarios "real" y virtual permiten matizar otras formas de comprensión de las problemáticas que demandan ser estudiadas cuando de educación virtual se trata.

Los documentos revisados interrogan el sentido de la educación hoy y ponen de presente sus implicaciones con "lo tecnológico", desde un espectro que procura trascender la mirada del objeto mismo en cuanto recurso, artefacto o dispositivo y en cuestionamiento de las relaciones entre las fuerzas socioculturales y las respuestas tecnológicas que "pretenden atender demandas sociales". Los autores coinciden en sus discusiones en advertir acerca de la

10 Ros (2004); Del Moral, Villalustre (2005); Mauri, Colomina (2007).

11 Gil, Feliu, Rivero (2003); Villalobos (2004); Rozo (2008). 
necesidad de reconocer la emergencia de la cultura digital demarcada por prácticas específicas que, de ninguna manera, excluyen las construcciones individuales y colectivas que se dan en el espacio físico (real), pero que contienen en sí mimas unas maneras de ser, estar y asumir su presencia y participación en el entorno red.

Como refiere el equipo de la UOC (Gil, Felliu, Rivero, 2003), "la generación educada en este inicio de siglo XXI también es audiovisual, pero lo que la caracteriza, es que emerge ya en el interior de una cultura digital, que, como señala Tapscott es una generación que llegará a la mayoría de edad bañada en bits". De este modo se subraya la necesidad de analizar la cultura de la actual generación: sus formas de relación, de comunicación; las ideas de futuro, de trabajo; sus deseos, sus identidades. Análisis realizados con la intención de actualizar los viejos problemas de una educación descentrada -entre otras- por las demandas de una sociedad posmoderna carente de certezas y por la adopción sin más de tecnologías y medios que facilitan el acceso a grandes volúmenes de información y desde donde se proponen otras formas de relación y construcción de conocimiento.

El mismo trabajo se ocupa de indagar por esas prácticas de los jóvenes por fuera de la institución escolar que aportan -desde experiencias no oficiales- a la construcción de conocimiento, a la configuración de identidades y a la producción de sentidos, significados y símbolos emergentes desde esa otra cultura y que, de manera indefectible, requieren ser considerados en propuestas de E. V.

Algunos de los hallazgos más importantes de ese estudio validan lo anterior: el encuentro de los jóvenes alrededor de las TIC en los cibercafés de Barcelona muestra cómo las prácticas de consumo, asociadas al tiempo de ocio "invertido" en estos lugares, van creando identidades y construcciones simbólicas: "la identidad se imagina más como una posibilidad, como algo que hay que construir en cada contexto, más que como algo predeterminado que ya viene dado". En las prácticas de producción, "se adoptan determinadas estéticas, formas de relación, músicas, argots, se apropian determinados espacios físicos (y virtuales)... la misma selección de actividades y de objetos produce identidad" que son consideras por los mismos jóvenes como prácticas poco útiles, poco valiosas. En la pretensión de trasladar estas prácticas a las formas de relación y de producción que se pretenden en la institución escolar, la práctica misma adquiere otro significado y pierde valor como lugar de encuentro, de significación, de construcción, de experimentación.
Entre tanto, el documento de Villalobos (2007) identifica la ruptura de la estructura lineal del texto y las oportunidades del lector en condición de autor-lector desde las posibilidades que facilita el hipertexto; características que, desde un enfoque socio-crítico, aportarían, a criterio del autor, al diseño de programas de E. V., en la consideración del proceso educativo como una permanente negociación de sentidos en contextos específicos y un reconocimiento de la construcción individual y colectiva -más que un fenómeno de recepción y repeticiónEl artículo analiza la conexión entre el hipertexto y los postulados pedagógicos de Paulo Freire, en tanto que se establece "una íntima relación entre las fuerzas socioculturales y las respuestas técnicas que surgen en el proceso dialéctico entre ellas", al tiempo que advierte cómo el hipertexto también podría ser empleado para reproducir modelos de educación transmisionistas cuando las posibilidades comunicativas y de construcción no se tienen en cuenta. De acuerdo con Villalobos,

[...]De acuerdo con el trabajo de Villalobos "el hipertexto como una estructura digital de múltiples lexías enlazadas entre sí, y que establecen relaciones intertextuales en diversos niveles de contextualización a través de medios verbales (signos de la escritura, la palabra hablada, etc.) y no verbales (imágenes y sonidos)”. "El hipertexto, confronta al lector con los múltiples posibles caminos que se abren con cada enlace. Le motiva a tomar conciencia del camino a seguir, le fuerza a convertir su trayecto en un quehacer, le convierte, en sujeto de su lectura: de espectador a creador". (Gómez Martínez, 2001).

En último lugar, Rozo (2008) da cuenta de la construcción colaborativa de la propuesta pedagógica para escenarios mediados tecnológicamente; propuesta que sirvió de base para el diseño de programas de posgrado en la Universidad Pedagógica Nacional. En ella se advierte la importancia de considerar los factores que inciden en las transformaciones socio-culturales propias del entorno digital; se presentan los aspectos centrales que el equipo de trabajo consideró para el diseño de la propuesta; y se reconocen aspectos valiosos al entender la virtualidad como potencia, la exploración de campos problemáticos -de acuerdo con Lévy y en función de lo virtual-como oportunidad de indagación, la reconfiguración en entornos que reflejan mutaciones constantes dadas por las formas como se habita la red y la emergencia de lenguajes y modos de comunicación y otras relaciones del espacio-tiempo que modifican, de manera sustantiva, los vínculos con la información y las maneras de producir conocimiento.

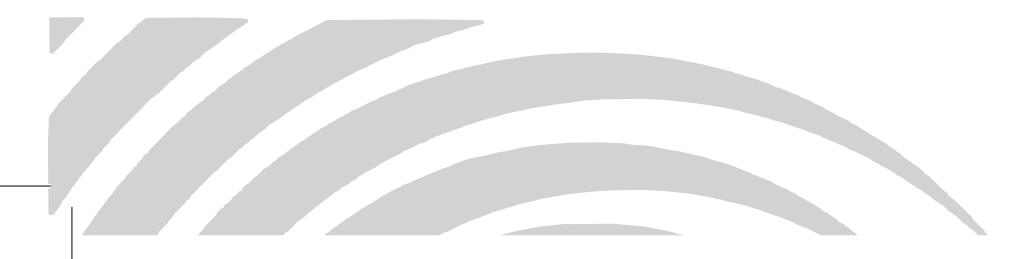


Los resultados de tal experiencia resaltan el sentido de la educación, el carácter de lo pedagógico, la importancia de re-conceptualizar lo tecnológico y la tecnología, la necesidad de considerar a quienes intervienen en el acto educativo desde espacios que median la relación pedagógica con recursos mediáticos y tecnológicos, el valor de la interacción como posibilidad de comunicación dialógica y la indefectible pertinencia de analizar el ambiente de la red como un escenario con características propias, modos de actuación y relación que retan y tensionan la relación educación-comunicación-cultura. Finalmente, el documento señala que la validación de aspectos sociales y culturales, la comprensión de las subjetividades que emergen en estos entornos y de las identidades distribuidas en la red, así como los cambios sustantivos en los procesos de comunicación tradicional, constituyen aspectos neurálgicos que obligan a repensar la educación.

\section{Conclusión}

Interrogarse acerca de cómo

"En el mejor de los casos, el maestro se vincula al diseño de los materiales que se disponen en el A.V.A; pero, por lo general, se constituye en parte de un eslabón de la cadena que fragmenta la "producción del servicio educativo". Proceso donde prima el interés por aumentar el número de estudiantes..." escenarios de saber pedagógico, el currículo como virtualidad en condición y capacidad de ser transformado y el trabajo en red como la construcción de colectivos, de sujetos de saber, en condición de apropiar y construir conocimiento.

Las experiencias analizadas permiten inferir que la relación entre quienes intervienen en el acto pedagógico, mediado por enfoques de lo virtual y vinculados a la idea de simulación, se presentan como "eficientes" en cuanto reproducen o simulan el espacio y las prácticas de aula, el currículo, los enfoques pedagógicos y de evaluación; valoración derivada de interpretar lo virtual como habitar la red. Así, las preguntas por la identidad, la subjetividad, las interacciones, las múltiples relaciones -comunicativas, con el conocimiento, de poder, etc.- $y$, en general, las formas de ser sujeto en la virtualidad son aspectos que se mantienen como constantes de reflexión e inciden en los desarrollos que cada vez más imbrican lo virtual y lo real; de este modo se alejan de la idea que confronta y antagoniza estos dos entornos.

Con alguna fuerza emergen trabajos que advierten de la "crisis de identidad" en la red como consecuencia, entre otros aspectos, de las relaciones particulares que allí tienen lugar, de las formas de comunicación, de legitimación, de estar presente a través de identidades distribuidas y de ser parte de la comunidad, del colectivo. Estas maneras de ser y habitar la red descentran el papel tradicional del maestro, quien debe asumirse de otra forma; en especial, porque en el "ambiente virtual" se disponen los contenidos, las actividades, los procesos de evaluación y su función se debate entre "acompañante, tutor, dinamizador, orientador". En el mejor de los casos, el maestro se vincula al diseño de los materiales que se disponen en el A.V.A; pero, por lo general, se constituye en parte de un eslabón de la cadena que fragmenta la "producción del servicio educativo". Proceso donde prima el interés por aumentar el número de estudiantes al delegar la responsabilidad con la producción, asimilación y apropiación de conocimientos a los diseños sofisticados y atractivos puestos en las plataformas. del papel de los maestros como actores políticos y constructores de sociedad, las tecnologías como 
De igual manera, la idea del "sujeto virtual" y sus múltiples relaciones permiten avizorar que se hacen necesarias otras comprensiones en las formas de aprender -y también de enseñar- cuando, quienes hacen parte activa de este proceso, desarrollan prácticas específicas de comunicación, de configuración socio cultural en red y de construcción colaborativa, por mencionar algunos de los factores explorados en el estudio.

Ahora bien, en la relación información-conocimiento se hace claro que, en las propuestas educativas mediadas tecnológicamente, se mantiene una conexión con los actores y los enfoques de ambientes educativos por cuanto, en la concepción de virtual asociada a la idea de red, las relaciones múltiples que se tejen entre quienes allí habitan son parte constitutiva de estos entornos. Con esta compresión, los trabajos analizados dan cuenta de las tensiones evidentes entre las concepciones de lo comunicativo y de lo educativo asociadas a la transmisión y a la validación de información. Tensiones en las cuales los recursos mediáticos y tecnológicos se constituyen en los principales mediadores del proceso formativo y, frente a otras apuestas que dan valor a la idea del autor-lector, reconocen la emergencia de lenguajes más visuales, hipertextuales y multimediales y consideran lo educativo como proceso de construcción. Estas maneras de aproximarse al problema de lo educativo en "entornos virtuales" orientan los diseños de los denominados AVA, OVA, EVA y, desde allí, se ponen en escena las concepciones que subyacen a lo educativo, lo comunicativo, lo tecnológico y lo filosófico.

\section{Bibliografía}

Benítez, R. (2000), "La educación virtual, desafío para la construcción de culturas e identidades”, en Congreso Proyección de la Integración Latinoamericana en el siglo XXI, [en línea], disponible en: www.umcc.cu/ boletines/educede/.../educacionvirtualcultura.doc, recuperado: 30 de agosto de 2009.

Castells, M. (1999), La era de la información, Vol. 1, Madrid, Alianza.

Coll; Mauri y Onrubia. (2006, octubre), "Análisis y resolución de casos-problema mediante el aprendizaje colaborativo" en Revista de Universidad y Sociedad del Conocimiento, [en línea], vol. 3, núm. 2, disponible en: http://www.uoc.edu/rusc/3/2/dt/esp/coll_mauri_onrubia.pdf, recuperado: 10 de junio de 2009.
En este sentido, se hace pertinente señalar la importancia que, desde los trabajos del grupo de Barcelona y algunos de la UOC, se otorga a las interacciones -entendidas como procesos comunicativos en los cuales la negociación de sentidos adquiere un valor trascendental cuando de construir conocimiento se trata-. En la misma línea, la idea del aprendizaje en red, colaborativo y cooperativo se muestra como opción de trabajo viable a la hora de abordar procesos de construcción; sin embargo, es preciso señalar que las tecnologías y los recursos -como el hipertexto- por si solos no determinan procesos de construcción ni de colaboración.

Finalmente, esta revisión de las investigaciones en materia de E. V. facilita la comprensión de problemáticas contemporáneas que cada vez requieren análisis más complejos, desde diferentes disciplinas. En especial cuando se habita una sociedad que se enuncia, sin más, como "sociedad de la información" y/o del "conocimiento", pero que desconoce las mutaciones socio-culturales que se dan entre los habitantes de la red, las fracturas en la relación espacio temporal que genera otras formas de aproximarse a la información y de construir conocimiento. Sociedad aislada, cada vez más, del análisis pendiente acerca de lo individual y lo colectivo en la red, lo público y lo privado y que cada vez fomenta más la adopción de tecnologías y modelos educativos carentes de reflexión, aislados del compromiso político y social que tiene la educación.

Correa Gorospe, J. M. (2005), "La integración de plataformas de e-learning en la docencia universitaria. Enseñanza, aprendizaje e investigación con Moodle en la formación inicial del profesorado" en Revista Latinoamericana de Tecnología Educativa, [en línea], 4 (1), pp. 37-48, disponible en: http://www.unex.es/ didactica/RELATEC/Sumario_4_1.htm, recuperado: 12 de junio de 2009.

Del Moral, P. y Villalustre, L. (2005, julio), “Adaptación de los entornos virtuales a los estilos cognitivos de los estudiantes. Un factor de calidad en la docencia virtual", en Revista de Medios y Educación, [en línea], núm. 26, disponible en: http://redalyc.uaemex.mx/ redalyc/src/inicio/ArtPdfRed.jsp?iCve=36802602, recuperado: 10 de junio de 2009.

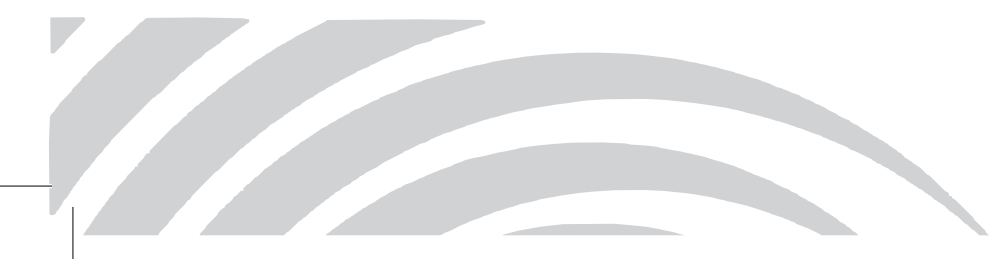


Flores, E. (2006), "Encontrando al profesor 'virtual'. Resultados de un proyecto de investigación-acción", en Revista Mexicana de investigación Educativa, [en línea], núm. 28, disponible en. http://www.comie.org. $\mathrm{mx} /$ documentos/rmie/v11/n28/pdf/rmiev11n28scB02n04es.pdf, recuperado: 26 de mayo de 2009.

Freire, P. (1980), Pedagogía del oprimido, Buenos Aires, Siglo XXI Editores.

Gallardo; Tarrandell y Negre. (2005), “Estudios de modelos organizativos en la enseñanza universitaria mediante entornos virtuales. EDUTEC '05”, en Congreso Internacional sobre Formación del profesorado y Nuevas Tecnologías. Santo Domingo (República Dominicana).

García Canclini, N. (2004), Diferentes, desigualesy desconectados. Mapas de interculturalidad, Barcelona, Gedisa.

Giesecke, M. (2009), En busca de ideales postipográficos, García, T. (Trad.), Alemania, Universidad de Erfurt.

Gil, A. et al. (2003), ¿Nuevas tecnologías de la información y la comunicación o nuevas tecnologías de relación? Niños, jóvenes y cultura digital, [en línea], disponible en: http://www.uoc.edu/dt/20347/, recuperado: 10 de junio de 2009.

Giroux, H. (2005), Estudios culturales, pedagogía crítica y democracia radical, Madrid, Popular. Colección Proa.

Giroux, H. y Mclaren, P (1998), Sociedad, cultura yeducación, Madrid, Miño y Dávila (edits.), Instituto Paulo Freire.

Ilvento, C. y Martínez, M. (2007), "Acerca $®$ de la construcción de imaginarios identitarios en el tutor virtual", en XII Congreso Internacional de Informática en la Educación, La habana, Cuba, [en línea], disponible en: www.informaticahabana.com/evento_virtual/files/ EDU016.doc, recuperado: 26 de mayo de 2009.

Kaplún, M. (1993), "Del educando oyente al educando hablante", en Revista Diálogos de la Comunicación [en línea], núm. 37, disponible en: http://www. dialogosfelafacs.net/dialogos_epoca/pdf/37-02MarioKaplun.pdf, recuperado: 6 de julio de 2009.

Lèvy, P. (1995), ¿Qué es lo virtual?, Barcelona, Paidos.

Langdon, W. (2000), "Los mitos ciberlibertarios y sus prospectos para la comunidad", en Revista Digital de Investigación y Nuevas Tecnologías, [en línea], núm. 4, disponible en: http://contexto-educativo.com. ar/2000/2/nota-1.htm, recuperado: 5 de febrero de 2010 .
Madrid, L. (1999), Sobre la dimensión pedagógica y política de la formación del profesorado [en línea], Disponible en: http://aufop.com/aufop/uploaded_files/ articulos/1224534248.pdf, recuperado: 1 de julio de 2009.

Martínez, T. y Maris, S. (2007), “Contigo en la distancia. La práctica tutorial en entornos formativos virtuales", en Revista de Mediosy Educación Universidad de Sevilla, núm. 29, [en línea], disponible en: http://redalyc.uaemex. $\mathrm{mx} /$ redalyc/src/inicio/ArtPdfRed.jsp?iCve=36802907, recuperado: 10 de junio de 2009.

Mauri T.; Colomina, R. y de Gispert, I. (2007), “Diseño de propuestas docentes con TIC en la enseñanza superior. Nuevos retos y principios de calidad desde una perspectiva socio constructivista", en Revista de Educación, [en línea], disponible en: http://www. ub.edu/grintie, recuperado: 10 de junio de 2009.

Mungaray, A. (2005), "Sujetos virtuales de conocimiento. Los retos de la información en el hipertexto", en Revista Electrónica de Investigación Educativa, 7 (1). [en línea], disponible en: http://redie.uabc.mx/ vol7no1/contenido-lagarda.html, recuperado: $10 \mathrm{de}$ junio de 2009.

Mungaray, A. (2007), "Algunas dimensiones de las redes de información y su impacto en las estructuras de conocimiento. Un marco conceptual", en IX Congreso $\mathrm{Na}$ cional de Investigación Educativa. México, [en línea], disponible en: http://www.comie.org.mx/congreso/ memoria/v9/ponencias/at04/PRE1178923671.pdf, recuperado 15 de junio de 2009.

Onrubia J., (2005), Aprender y enseñar en entornos virtuales: actividad conjunta, ayuda pedagógica y construcción del conocimiento, [en línea], disponible en: http://www.um.es/ead/red/M2/conferencia_onrubia.pdf, recuperado: 26 de mayo de 2009.

Ortega, P. (2008), "Hacia una comprensión sobre la pedagogía crítica”, en Rozo et al., Contextos y pretextos sobre la pedagogía, Bogotá, Universidad Pedagógica Nacional, Colección Instituto de Tecnología Abiertas en Educación, ITAE.

Pérez, J. (2003), Plataformas digitales y sus fracturas pedagógicas, [en línea], disponible en: http://revistas.ucm. es/edu/11302496/articulos/RCED0303220563A. PDF, recuperado: 26 de mayo de 2009. 
Ros, A. (2004), "La verdadera apuesta del aprendizaje virtual. Los aspectos sociales del e-learning”, en Revista de Universidad y Sociedad del Conocimientos (RUSC), [en línea], disponible en: http://www.uoc. edu/rusc/dt/esp/ros0704.pdf, recuperado: 15 de junio de 2009.

Rozo, C. (2008), “Propuesta pedagógica para educación a distancia con mediación tecnológica”, en I Encuentro Internacional en Educación Virtual, Bogotá, Corporación Universitaria Iberoamericana-Colciencias.

Rozo et al. (2008), Contextos y pretextos sobre la pedagogía, Bogotá, Universidad Pedagógica Nacional, Colección Instituto de Tecnología Abiertas en Educación, ITAE.

Rueda, P. (2004), “Sociedad red, Cultura, tecnología y pedagogía crítica", en X Conferencia de Sociología de la Educación [en línea], disponible en: http://www. uv.es/ jbeltran/ase/textos/pascual.pdf, recuperado: 1 de julio de 2009.

Saldarriaga, O. (2006), "Pedagogía, conocimiento y experiencia. Notas arqueológicas sobre una subalternización", en Revista Nómadas, núm. 25, pp. 98-108.

Salinas; Negre y Gallardo (2006), Modelos didácticos en entornos virtuales de formación: identificación y valoración de elementos y relaciones en los diferentes niveles de gestión, [en línea], disponible en: http:// gte.uib.es/publicacions/edutec06.pdf, recuperado: 28 de agosto de 2009.
Sarlé, P.; Rodríguez, I., y De Angelis, S., (2008), Proyecto Margarita. "Una comunidad virtual de maestros en Co formación”, en Revista Razón y Palabra, núm. 63, [en línea], disponible en: http://www.razonypalabra. org.mx/n63/psarle.html, recuperado: 26 de mayo de 2009.

Serres, M. (2001, 18 de junio), "Lo virtual es la misma carne del hombre”, en Le Monde, Paris.

Silva, J. (2005), "Las interacciones en un entorno virtual de aprendizaje para la formación continua de docentes. Una experiencia Chilena con docentes de enseñanza primaria”, en Sánchez, J. (edit.), Nuevas Ideas en Informática Educativa, Santiago: LOM Ediciones, pp. 192-201.

Soby, M. (2000), Identy and Learning in Cyberspace, en Sandbothe, M. y Marotzki, W. (edits.) Subjektivität und Öffentlichkeit. Kulturwissenschaftliche Grundlagenprobleme virtuellen Welten, Colonia, Herber von Halem.

Velandia, A. (2005), Hacia un modelo de universidad pública en la virtualidad, el caso de la UAM-X, [en línea], disponible en: http://redalyc.uaemex.mx/ redalyc/src/inicio/ArtPdfRed.jsp?iCve $=34004405$, recuperado: 10 de junio de 2009.

Villalobos, J. (2004), "Paulo Freire. Pedagogía e hipertexto", en Educere, La Revista Venezolana de Educación, [en línea], disponible en: http://redalyc.uaemex.mx/ redalyc/src/inicio/ArtPdfRed.jsp?iCve=35602608, recuperado: 10 de Junio de 2009. 